\title{
A mathematical model for agroforestry optimization
}

\author{
${ }^{1}$ CARLOS RODRÍGUEZ LUCATERO, ${ }^{2}$ MARCELO OLIVERA VILLAROEL, \\ ${ }^{3}$ PAOLA OVANDO \\ ${ }^{1}$ Departamento de Tecnologías de la Información \\ Universidad Autónoma Metropolitana Unidad Cuajimalpa, MEXICO \\ ${ }^{2}$ Departamento de Teoría y Procesos del Diseño \\ Universidad Autónoma Metropolitana Unidad Cuajimalpa, MEXICO \\ ${ }^{3}$ Research Staff Member Spanish Council for Scientific Research, SPAIN
}

\begin{abstract}
In the present article, we will describe some extensions of an agroforestry model that has been proposed and computationally implemented in [7]. Our generalizations consist of the inclusion of two additional species of tree, one culture, and a declaration of regeneration tours as variables definable by us as a parameter and the weight allocation by rentability of the treeless soil utilization as well as an exhaustive exploration of the different soil utilization scenarios in order to obtain the one who gives the best economic performance.
\end{abstract}

Key-Words: Optimal control, Mathematical modeling, Agroforestry, Environmental sustainability

Received: April 8, 2021. Revised: January 20, 2022. Accepted: February 10, 2022. Published: March 2, 2022.

\section{Introduction}

The problem of forest regeneration in lands whose land use is shared between forest preservation, agriculture, or cattle grazing has been one of the great challenges of forest preservation. Such a situation is the case of the Dehesas in Spain where private landowners who make mixed use of the land are faced with the dilemma of making sustainable use of their lands in environmental terms and, on the other hand, exploiting whatever resource is economically attractive. In this last sense, various initiatives can be observed in Europe to promote the forestry development of these multiple-use lands through government subsidies to stimulate the planting of trees. The development of wooded areas in these lands in private hands can turn them into spaces for ecotourism or even depending on the type of tree planted; it could be used to produce cork such as cork oaks or produce acorns to feed pigs whose meat is highly valued for its quality as would be the case of holm oaks. In the review that we have carried out on this topic, we can mention the work carried out in the case of Dehesas in Spain by the authors of [4, 5, 7]. In this article, we are inspired by the work carried out by [7] where the problem of economic and sustainable optimization of pastures in the Andalusian region in southern Spain is studied. In this article, he proposes a model of economic and agroforestry optimization where land use for grazing, agriculture, and sowing of oaks and cork oaks is combined. In this mixed use of the land that they carry out in the article mentioned above, they define some rules of rotation of both pasture crops and tree renewal based on the lifetime of each tree species and also taking into account the natural reforestation specific to each species. The distribution of wooded and nonwooded areas from which the optimization model is started is introduced as initial conditions. Once some of these parameters have been defined, a linear optimization is carried out, taking into account the restrictions imposed by the needs of crop rotation as well as the search for obtaining economic profit. Of course, one of these restrictions is not exceeding the limit of land available. We have included the details of the model exposed in [7] as well as a description of it in the section 9 of this article for the purpose of completeness of the exposition of our model. Based on the initial distribution of surfaces as well as non-negativity restrictions of the variables involved, a MATLAB linprog toolbox for linear optimization routine is called that produces the decisions that will be made at each time step, and this information feeds the dynamic model that describes the behavior over time of the system from the application of the decisions calculated by the linear optimizer mentioned above. The time window used in both the [7] model and ours is 0 to 100 years. The solution to the forest optimization problem raised in [7] is approached as an optimal control problem in discrete time and finite time horizon and that describes the agroforestry decision processes by social and private agents in a pasture system. In this dynamic optimization model, laws of motion of the states are defined during the time horizon, control variables and conditions of non-negativity of these, global restriction of resources, as well as artificial and natural regeneration restrictions of adult trees that are useful for 
the definition of physical and legal restrictions in each case study addressed in our research. Some additional restrictions relative to the planting of holm oaks and cork oaks on bare ground are added for each specific case, which guides forest management to a steady state. Similarly, an objective function is defined that represents the variable of income at net present value at a fixed horizon for each of the Dehesa farms studied in [7]. The same authors of [7] define a gradient vector of the objective function that allows them to understand the operation of the proposed dynamic optimization program, and from the expressions that appear in said gradient vector, the linear nature of the decision problem can be observed. In the model that we have just described very quickly in the previous paragraphs, a very complete analysis of the income or earnings of a farm owner is made both in scenarios where there is public intervention through subsidies and incentives to friendly developments with the environment, as in scenarios where there is no public intervention. In that sense, this work is very complete. However, the rules that are defined for the use of the land for reforestation purposes or for agriculture are carried out by defining very specific rules to the particular needs of each of the farms studied, which seems to us to be a limitation of this model. Another limitation of the model mentioned above is that it only works with two tree species (holm oak and cork oak). On the other hand, the optimization is carried out from a single scenario of initial distribution of crops in bare soil. Due to these limitations in the model proposed in [7], we propose a generalization of the previous model by adding a greater number of tree species, discarding the Adhoc rules of crop distribution in bare soil and evaluating all possible crop distributions on bare ground to choose the scenario that maximizes income. The latter can generate degenerate cases in which the global resource restriction is violated and that we eliminate after processing. On the other hand, this exhaustive test of the possible initial distributions can be costly in terms of calculation time, which we try to alleviate by means of a random sampling of the space of possible initial distributions of bare soil. This model expands and refines [5] by allowing a more flexible framework for land use replaceability and tree species selection. This approximation then allows us to examine in discrete time the composition and distribution of managed forest, abandoned forest, and non-forest uses at the farm level, which characterizes private land holdings in the region that is the empirical focus of our paper. This type of models has been previously applied to analyze efficient forest investment decisions through afforestation programs [22] forest management investments [5] and forest management decisions, such as thinning [20],[21]. Optimal control techniques in dis- crete time have also been applied to solve farmland decision problems, whereas the farm owner or holder has a preference to conserve forest areas within the farm [3],[19]. The contribution of our paper is as follows. Our primary focus contributes to understanding how different initial land use distributions and environmental conditions affect private actors' investment decisions in forest management and afforestation. These changes in the distribution, in turn, throws light on how these decisions affect the longer-term provision of ecosystem services and (relatedly) forest ecosystem asset values over time [24],[25]. That is what our contribution in this article consists of, which will be described in the following sections of this work.

\section{Optimal management of forest systems based on environmental economic behavior}

In this article we develop a framework of economic analysis for agroforestry systems by implementing of a bio-economic mathematical model based on a dynamic optimization system inspired in the work developed by [7] and [22]. For this reason we use as object of evaluation of the optimization system, the rent at present value in perpetuity of a typical farm. The model allows to see the impact on the conservation of forest areas through the implementation of policies of subsidies to forest products, the payment of environmental services, the subsidies of the generation of commercial plantations and the effects of livestock control without restrictions in the systems Forestry [8], being able to extrapolate to different types of ecosystems as long as the level of analysis is a farm type. The calculation of the annual income of the farm type at present value considers an infinite horizon time whereby it develops a process of normalization for generating an average farm. The optimization model uses as methodology the resolution of an optimal control problem in discrete time following the research line of [1],[3], [7], [19] considering the decision of the agents that manage a typical farm with forestry systems within their production system and the preference to conserve forested area on the farm. It is considered that the income of the farm includes a function of the price of the land determined by the age of the trees and the agricultural activities developed on the farm, being a substitute of the price estimated by the market of forest systems. It is assumed that decision-makers are maximizers of the economic rent [4], [7]. In order to obtain the optimal income of the farm analyzed, different land use options are categorized in order to maximize the income at present value of the integrated management of the farm. In the model proposed in [7] it is assumed that 
the owner decides between the different land use options from the initial distribution that faces as owner. That is, it decides which should be the percentage of land allocated to different forest and agricultural options. Such decisions are taken in concordance with the costs and long-term benefits that offer the farm size. One of the possible options is the abandonment of the farm in the long term. The model considers that the different uses of the land are replaceable between them, as long as the cost benefit of the chosen activity pays the replenishment and opportunity costs of the activities to be replaced, considering the substitution between more than two forest species, thus expanding the base model developed by [7] That is, the objective function of the analysis framework is a decision model where the renewal of the forest system associated with the farm, that has as options the natural renewal with associated management, the artificial renewal with the development of plantations or the abandonment of the systems forestry with the introduction of livestock systems without associated forest management. Unlike [7] the model considers non-linear cost systems in the development of the income models of the type farms analyzed, considering the substitution of more than two forest species in the farm as well as pastures and agricultural systems. Moreover, in contrast to the works of [11] and [22] the farms abandonment is considered as a plausible option to the agro-forestry management model. Within the forest management process of the analysis framework is considered a decision already made in advance, optimal shifts according to the forest species existing in each farm, that it is considered an exogenous decision to the process of optimal forest control, solving the problem of [10]. The model's objective is to choose the type of renewal of forest systems associated with the farm through the selection of optimal sequences of reforestation, forest plantations, and natural regeneration of existing adult trees, abandonment of the forest or the implementation of new agricultural areas of pastures. The final goal is to offer an optimal distribution of land use within the farm, each time one of the cutting shifts of the existing forest species is effective. The model takes into account a typical farm considering the following restrictions and assumptions to obtain the annual income of the enterprise according to the type of renewal of the forest systems associated with the farm:

1. The number of commercial forest species on the farm

2. Production cycles of each forest species. Theoretical growth rate of the commercial forest species

3. The land use of the farm including conserva- tion areas, agricultural (cereal crops, fruit trees, grassland among others), forest management and abandoned or dismantled areas

4. Age of the existing trees on the farm

5. Depending on the case, the management of mature forests (steady state) or forest plantations in their initial stages of growth can be considered.

6. Types of management of forest systems, if it is a natural regeneration or a commercial plantation

7. Profits from agricultural activities are considered part of the land use income

8. Livestock activities directly affect the processes of natural regeneration

9. The costs and benefits of the production of forestry and agricultural species in the long term are considered, as well as the provision of other types of services associated with forest systems and the farm as recreational and hunting activities.

10. Forestry activities include the collection of nontimber and timber products

11. Subsidies are considered by productive systems, that is, forestry, agriculture and/or conservation

12. The costs and sale prices within the production system are normalized to a reference year to avoid short and medium term distortions such as inflationary shocks or the exchange market. That is, it is considered a constant price for the development of the analysis.

13. The model considers both positive and negative price shocks

14. The model considers that the farm is taking market prices and cannot affect them in the short or long term

\section{Different discount rates are considered}

In our model we call this initial distribution of land use options scenario. Our model is a generalization of the model proposed in [7] because we generate each possible scenario or initial land distribution options and for each possible scenario we apply the same kind of decision optimization performed in [7]. We obtain and record for each scenario its economic utility. After processing all the possible scenarios we select the one that has the higher economic utility. 


\section{Current dynamics of European forest systems}

The current dynamics of European forest systems cannot be understood without the interference of human activities in the forest environment; since the composition and structure of the landscape is conditioned to the interactions of the existing conditions in space and the use of space by humans [23]. The use of European forest systems traditionally seeks to effectively use the resources available in the environment. Thus, the current distribution conditions of agricultural activities with the efficient use of pastures and land suitable for cultivation are associated with the composition of forest systems for the extraction of timber and non-timber resources; [12] generating cultural landscapes with its own dynamics of biodiversity and economic productivity [6] An example of this, the cork oak forests and the combination of cereal crops and pastures and hunting activities in the region. The composition and structure of forests are strongly associated with the profitability of the economic and of each region's economic and social dynamics so that the landscape units of these systems are always in constant movement. This is evident in the last 50 years with the demographic changes of rural systems in Europe, where there is an abandonment of rural activities and there is an ageing of population structures, an example is the abandonment of farmland, or the raising of sheep and goats [5], [15]. The change in demographic composition has had an impact on the amount of energy contributed by humans to their environment, leaving aside the agricultural and forestry exploitation and with it a change in the structure of the landscape [23]. Changes in this artificial composition of the environment have many consequences among the most relevant: a loss of value in the economic production of forest systems for their owners and therefore an abandonment of pre-existing forest management; loss and spaces with very important cultural, economic and social values; losses of crops and pastures due to the increase in transitional spaces of thickets and groves, although this has a lower erosion rate, higher water quality in rivers and reservoirs and an unclear result on the gain and loss of biodiversity according to the stage of forest succession in which the abandoned space is located [17], [18]. The long-term effect over outgoing is the gain inhomogeneity of forest structure and a lower risk of fires, although in the process of forest succession can be generated colonization of shrub species, generalist arboreal, and pyrophytes that make the landscape lose its economic and environmental value and increase their risk of fires [2] In this sense, the European Union develops a series of public policies for forest management, which is summarized in Rural development pol- icy, Protection against fire and air pollution, Conservation of biodiversity, adaptation to climate change, Competitiveness of forestry investigation. The actions are on outgoing of these policies include subsidy programs for forest plantations, payment of environmental services, incentives for the conservation of non-timber extractions such as cork and acorn collection and non-extractive activities such as hunting activities among others [9]. The changes described both in the demographic composition of forest regions, local economic incentives and regions make forest management a complex issue, which must be faced by decision-makers of public policies as well as owners of farms with forestry systems throughout the country. In this sense, develop a framework of analysis that models the optimal behavior of a forest state, based on the economic benefits of forest management, is necessary to see the composition and distribution of forest systems. In the case of the study, the analysis will focus on optimizing the management of a typical forest state. We develop an economic analysis framework applied to agroforestry systems through the implementation of a bioeconomic model based on a dynamic optimization system. This type of models have been previously applied to analyze optimal forest investment decisions, though afforestation programs [22], and or forest management investments [5],[7]. A number of studies apply optimal control techniques in discrete time to solve farmland decisions problems whereas the farm owner or holder has a preference to conserve forest areas within the farm [3]. This model expands and refines [5] optimal control model allowing for a more flexible optimization framework in terms of land use replaceability and species selection. More specifically, our model includes two more forest species, considering in total four forest species that can be selected out of eight possible options at each farm. On the other hand, forest species can be substituted at the same land plot, for more profitable species as existing trees reach their rotation age. This latter change respect to [5] model is possible as our models allows for the competition of the four forest species for land that is available for afforestation/reforestation, which in turn accounts for both non-forest land uses (i.e. treeless shrubs, pastures, crop lands and other) and abandoned forest. The management of forest represents a complex problem, since forest can be considered as renewable resources, at the time they can be exhausted in order to satisfy other land use demands, such as food production or land development. A way to achieve an equilibrium point between these opposites forces is to propose models that include other forest benefits, that are normally not considered in conventional economic analysis. To this end we use mathematical optimization tools based on the theory of optimal control. 


\section{Dynamic optimization}

We are going to model our forestry problem under the methodology of dynamic optimization.

Our basic hypothesis is:

Uniqueness: although each of the phenomena analyzed ( land use on farms ) have similar behaviors and react in a similar way to external and internal stimuli as can be for instance, subsidies and taxes, change in relative prices of forest, agricultural and livestock products that determine the change in land use on farms, but different departure point. Since conditions of distribution of land uses are very different in each farm, being conditioned by geographic, climatic and historical factors, we consider that we can apply linear optimization processes to our problem. Our main goal is to optimize the incomes that are influenced by the changes in land uses. There are two main types of land use in the farms:

- Woodlands

- Treeless

Woodlands are classified by commercial species that generate income to the owners of the farm.

Commercial species have two handling options:

- Natural character where species regenerate naturally, which mean that the species meets its cycle of natural reproduction and drive conditions that allow their reproduction of the species in a controlled manner, giving the conditions for the prevalence of the species being replaced by systems of secondary forests or thickets systems, which are extremely expensive to replace other land use. So, once the forest is replaced by scrub systems there is no change in land use.

- The other option is artificial character where the species are planted and managed in a controlled manner within the farm

There are some conditions that have to be taken into account within the process

- We can not have more land than the one provided from the beginning.

- There are no negative soil quantities

- There are substitution between land uses, but this substitution is between woodlands and treeless uses depending on the profitability of each land use.

- There is an additional limit given in handling land use scrub, scrub arising from the abandonment of trees that meet their natural cycle and are not given the conditions for the prevalence of the species being replaced by systems of secondary forests or thickets systems, which are extremely expensive to replace other land use. So, once the forest is replaced by scrub systems no change in land use.

\section{Optimal control Model description}

In this section we are going to describe the mathematical tools used in the model proposed in [7] as well as in our model. Both models are discrete-time finite temporal horizon optimal control models. Under this decision process, the agents try to maximize the present value profit corresponding to the average hectare of grassland and can adapt each year his forest management schedule by means of artificial plantations of trees on treeless soil and by natural regeneration of existing adult trees. The Matlab code used for implementing both models admit a maximal temporal horizon lower than the critical regeneration age from artificial planted blockhead whose value is $T<142$ years. In the following subsections we will describe the details of the mathematical model and the corresponding implementation in Matlab. For the sake of completeness the datails of the model proposed in [7] are included in the section 9 of the present article.

\subsection{Control vectors of our model}

The optimization process of the decisions taken by the agents involved in the agroforestal process follow the same procedure of the agents in model proposed in [7], but we have added in our model two more control vectors in order to include two new types of tree, the Stone Pine tree and eucalyptus. The control vectors are defined as follows:

$$
z_{t}=\left(\begin{array}{c}
n_{i, t}+n_{i, t}^{a} \\
0 \\
0 \\
\vdots \\
-n_{i, t} \\
\vdots \\
0 \\
0
\end{array}\right) z_{a, t}=\left(\begin{array}{c}
t_{i, t} \\
0 \\
0 \\
\vdots \\
-n_{i, t}^{a} \\
\vdots \\
0 \\
0
\end{array}\right)
$$

$$
v_{t}=\left(\begin{array}{c}
n_{s, t}+n_{s, t}^{a} \\
0 \\
0 \\
\vdots \\
-n_{s, t} \\
\vdots \\
0 \\
0
\end{array}\right) v_{a, t}=\left(\begin{array}{c}
t_{s, t} \\
0 \\
0 \\
\vdots \\
-n_{s, t}^{a} \\
\vdots \\
0 \\
0
\end{array}\right)
$$




$$
w_{t}=\left(\begin{array}{c}
n_{p, t}+n_{p, t}^{a} \\
0 \\
0 \\
\vdots \\
-n_{p, t} \\
\vdots \\
0 \\
0
\end{array}\right) w_{a, t}=\left(\begin{array}{c}
t_{p, t} \\
0 \\
0 \\
\vdots \\
-n_{p, t}^{a} \\
\vdots \\
0 \\
0
\end{array}\right)
$$

$$
r_{t}=\left(\begin{array}{c}
n_{e, t}+n_{e, t}^{a} \\
0 \\
0 \\
\vdots \\
-n_{e, t} \\
\vdots \\
0 \\
0
\end{array}\right) r_{a, t}=\left(\begin{array}{c}
t_{e, t} \\
0 \\
0 \\
\vdots \\
-n_{e, t}^{a} \\
\vdots \\
0 \\
0
\end{array}\right)
$$

Where $n_{p, t}$ and $n_{e, t}$ represent de Stone Pine tree and Eucalyptus surface naturally regenerated by natural planted trees of such species, $n_{p, t}^{a}$ and $n_{e, t}^{a}$ represent de Stone Pine tree and Eucalyptus surface naturally regenerated by artifially planted trees of such species, $t_{p, t}$ and $t_{e, t}$ represent the surface reforested with Stone Pine trees and Eucalyptus by humans. The rest of the variables still represent the surfaces mentioned in [7] model. The details of this model can be consulted in the section 9 of the present article.

Concerning the utilization of the deforested surface of land the previous model due to [7], the author imposed as a constraint just allowing one tree reforesting species for each deforested type of surface of a particular farm land. For avoiding these ad-hoc rules of reforestation that depended on the particular farm $j$. We eliminated the farm numbering unlike what is done in [7] model where there are 4 different types of farm and in each case one or two species of tree can be sown and in some cases only one type of tree can be sown in a specific treeless terrain type. See the equation 23 in section 9 of the present article where we describe the precedent model of [7]. From our point of view this was a very unnatural constraint and we modified this criteria allowing to reforest these surface by any species of tree using a weighted asignation of surface to each species that depends on their corresponding economic rentability.

$$
u_{t}=\left\{\begin{array}{l}
\left(\left(\xi_{t}-\left(t_{i, t}+t_{s, t}+t_{p, t}+t_{e, t}\right) / 4\right.\right. \\
\left(\xi_{t}-\left(t_{i, t}+t_{s, t}+t_{p, t}+t_{e, t}\right), t_{s, t}\right) / 4 \\
\left(\xi_{t}-\left(t_{i, t}+t_{s, t}+t_{p, t}+t_{e, t}\right) / 4\right. \\
\left(\xi_{t}-\left(t_{i, t}+t_{s, t}+t_{p, t}+t_{e, t}\right) / 4\right.
\end{array}\right.
$$

Where $\xi_{t}$ is a transition function of soil utilization and is calculated as follows

$$
\xi_{t}=\left\{\begin{array}{l}
x_{320, t-1}+x_{320, t-1}^{a}+s_{196, t}+s_{194, t}^{a} \\
+p_{320, t-1}+p_{320, t-1}^{a}+e_{320, t-1}+e_{320, t-1}^{a}
\end{array}\right.
$$

\subsection{The state vector movement law of our model}

Introducing the new state variable as well as the new control vectors the movement law equations that describe the temporal evolution of the controlled state variables are the following:

$$
\left.\begin{array}{l}
x_{t+1}=T x_{t}+z_{t+1} \\
x_{a, t+1}=T x_{a, t}+z_{a, t+1} \\
p_{t+1}=Q p_{t}+w_{t+1} \\
p_{a, t+1}=Q p_{a, t}+w_{a, t+1} \\
e_{t+1}=R e_{t}+r_{t+1} \\
e_{a, t+1}=R e_{a, t}+r_{a, t+1} \\
s_{t+1}=A_{n} s_{t}+v_{t+1} \\
s_{a, t+1}=A_{a} s_{a, t}+v_{a, t+1} \\
d_{t+1}=d_{t}+u_{t+1}
\end{array}\right\}=\forall t \in[0, T-1], T<\infty
$$

Where $T, A_{n}$ and $A_{a}$ have the same meaning of the one in the model proposed in [7] and where the matrix $Q$, and $R$ are the transition matrix, corresponding to stone pine and eucalyptus reforesting land and where the elements are equal to zero excepted the elements just under the main diagonal whose values are equal to 1 . The dimensions of $Q$ and $R$ are $321 \times 321$.

\subsection{The control variable constrains of our model}

The global resource restriction that establish that the sizes of each farm stay constant, is now expressed as follows:

$$
\begin{aligned}
S_{j}= & i\left(T_{2}\right) \cdot\left(x_{t}+x_{a, t}\right)+i\left(T_{6}\right) \\
& \cdot\left(p_{t}+p_{a, t}\right)+i\left(T_{4}\right) \cdot s_{t} \\
& +i\left(T_{4}\right) \cdot s_{a, t}+i\left(T_{8}\right) \cdot\left(e_{t}+e_{a, t}\right)+ \\
& i(4) \cdot d_{t} \\
& \forall j \in\{1, \ldots, 4\} \text { and } \forall t \in\{1, \ldots, T\}
\end{aligned}
$$

Where $i(n)$ is a row vector of ones of dimension $n$ and $S_{j}$ is the productive agricultural surface of farm 
$j$ for each $j \in\{1, \ldots 4\}$. Additionally some constrains regarding the natural regeneration process are imposed in such a way that the surface to be regenerated cannot be greater than the surface of trees in crital age. These constrains are expressed as follows:

$$
\left.\begin{array}{l}
n_{i, t} \leq x_{249, t-1} \\
n_{i, t}^{a} \leq x_{249, t-1}^{a} \\
n_{s, t} \leq s_{143, t-1} \\
n_{s, t}^{a} \leq s_{141, t-1}^{a} \\
n_{p, t} \leq p_{249, t-1} \\
n_{p, t}^{a} \leq p_{249, t-1}^{a} \\
n_{e, t} \leq e_{249, t-1} \\
n_{e, t}^{a} \leq e_{249, t-1}^{a}
\end{array}\right\}=\forall t \in[0, T-1], T<\infty
$$

By other side, the author of [7] mentioned that given the initial distribution of soil utilization, it can be observed that very big portions of land in each farm are available for being reforested. Given this situation, it can happen that the reforestation resources can be exhausted in one period of decision. For avoiding this case some constraints imposed on the number of reforestation hectares should be compliant with the spanish forestry and public laws. The corresponding proposed upper bounds are represented by the following inequalities:

$$
\begin{aligned}
& n_{i, t}+n_{i, t}^{a}+t_{i, t} \leq \frac{S_{i, j}^{n}}{250} \\
& \forall t \in[0, T-1], T<\infty \\
& n_{s, t}+n_{s, t}^{a}+t_{s, t} \leq \frac{S_{i, j}^{n}}{144} \\
& \forall t \in[0, T-1], T<\infty \\
& n_{p, t}+n_{i, t}^{p}+t_{i, t} \leq \frac{S_{i, j}^{n}}{250} \\
& \forall t \in[0, T-1], T<\infty \wedge j \in\{1, \ldots, 4\} \\
& n_{e, t}+n_{i, t}^{e}+t_{i, t} \leq \frac{S_{i, j}^{n}}{250} \\
& \forall t \in[0, T-1], T<\infty \wedge j \in\{1, \ldots, 4\}
\end{aligned}
$$

\subsection{Control Optimization Objective function of our model}

The objective function of a control optimization model represents the base on which a deciding agent takes decisions. In the case of this agroforestry problem the function whose value is maximized is a cubic polynomial that evaluates the profit net present value of the reforested land depending on the soil utilization. This function has the following mathematical form:

$$
\begin{array}{ll}
f_{i}=a_{i} \tau^{3}+b_{i} \tau^{2}+c_{i} \tau+d_{i} & \forall \tau \in\{0, \ldots, 320\} \\
f_{s}=a_{s} \tau^{3}+b_{s} \tau^{2}+c_{s} \tau+d_{s} & \forall \tau \in\{0, \ldots, 196\} \\
f_{p}=a_{p} \tau^{3}+b_{p} \tau^{2}+c_{p} \tau+d_{p} & \forall \tau \in\{0, \ldots, 320\} \\
f_{e}=a_{e} \tau^{3}+b_{e} \tau^{2}+c_{e} \tau+d_{e} & \forall \tau \in\{0, \ldots, 320\}
\end{array}
$$

Holm oak blockhead stobe pine eucalyptus

Based on these functions it can be calculated the land price for hectare row vectors for each type of planta- tion and stored in the following vectors:

$$
\begin{aligned}
& f_{i}=\left(f_{i}(0), \ldots, f_{i}(320)\right) \\
& f_{p}=\left(f_{p}(0), \ldots, f_{p}(320)\right) \\
& f_{s}=\left(f_{s}(0), \ldots, f_{s}(196)\right) \\
& f_{s, a}=\left(f_{s}(0), \ldots, f_{s}(196)\right) \\
& f_{d}=\left(p_{c t}, p_{p}, p_{m}, p_{o}\right)
\end{aligned}
$$

For enabling the agents to take decisions, it can be calculated the level of annual return vectors using diferent costing methods for each kind of soil utilization. The following row vectors store this information:

$$
\begin{aligned}
& y^{i, n}=\left(y_{0}^{i, n}, \ldots, y_{320}^{i, n}\right) \\
& y^{i, a}=\left(y_{0}^{i, a}, \ldots, y_{320}^{i, a}\right) \\
& y^{p, n}=\left(y_{0}^{p, n}, \ldots, y_{320}^{p, n}\right) \\
& y^{p, a}=\left(y_{0, a}^{p, a}, \ldots, y_{320}^{p, a}\right) \\
& y^{s, n}=\left(y_{0}^{s, n}, \ldots, y_{1, n}^{s, n}\right) \\
& y^{s, a}=\left(y_{0}^{s, a}, \ldots, y_{194}^{s, a}\right) \\
& y^{e, n}=\left(y_{0}^{e, n}, \ldots, y_{320}^{e, n}\right) \\
& y^{e, a}=\left(y_{0}^{e, a}, \ldots, y_{320}^{e, a}\right) \\
& y^{s, d}=\left(y_{c t}^{s}, y_{p}^{s}, y_{m}^{s}, y_{o}^{s}\right)
\end{aligned}
$$

Assuming that the deciding agent maximizes the finite horizon net present value of the profit associated to the average hectare of the farm $j$, the objective function gets the following form:

$$
\begin{aligned}
F_{j}= & \frac{1}{S_{j}}\left\{\sum _ { t = 0 } ^ { T } \delta ^ { t } \left[y^{i, n} x_{t}\right.\right. \\
& +y^{i, a} x_{a, t}+y^{s, n} s_{t}+y^{s, a} s_{a, t}+y^{p, n} p_{t} \\
& \left.+y^{p, a} p_{a, t}+y^{q, d} d_{t}\right] \\
& -\left[f_{i}\left(x_{0}+x_{a, 0}\right)+f_{s} s_{0}+f_{s, a} s_{a, 0}\right. \\
& \left.+f_{p}\left(p_{0}+p_{a, 0}\right)+f_{d} d_{0}\right] \\
& +\delta^{T}\left[f_{i}\left(x_{T}+x_{a, T}\right)+f_{s} s_{T}\right. \\
& \left.\left.+f_{s, a} s_{a, T}+f_{p}\left(p_{T}+p_{a, T}\right)+f_{d} d_{T}\right]\right\} \\
& q= \begin{cases}i \text { (ilex) } & \forall j \\
p \text { (pine) } & \forall j \\
e \text { (eucalyptus) } & \forall j \\
s \text { (suber) } & \forall j\end{cases}
\end{aligned}
$$

\subsection{Our model control optimization}

Finally the control optimization problem can be written as the maximization of the objective function as follows:

$$
\max _{\left\{n_{i, t}, n_{i, t}^{a}, n_{s, t}, n_{s, t}^{a}, n_{p, t}, n_{p, t}^{a}, t_{i, t}, t_{s, t}\right\}_{t=1}^{t=T}} F_{j}
$$

subject to: equations $(25)(26)(27)(28)(29)$ and the given intial conditions $x_{0}, x_{a, 0}, s_{0}, s_{a, 0}, p_{0}, p_{a, 0}, d_{0}$ as well as the time horizon $T$.

\section{Matlab implementation of our model}

As was mentioned in section 1 our model differs from the model proposed in [7] in two ways. The first is the 
introduction of an additional tree species, the stone pines. The details of our mathematical model are described in section 5. We also mentioned at the final part of the section 1 that our Matlab implementation generalizes the model of [7] in the sense while this model perform an optimization of the decision process just over one fixed scenario, our proposal first generate all the possible scenarios, then process all them in order to calculate for each one their present value profit and finally extract the scenario with respect to the obtained present value profit. In this way our model performs two kind of optimization, the first is over the decision process in each scenario and the second is on the selection of the scenario that has the best economic performance. The steps followed by our model can be expressed algorithmically as follows:

1) Table_of_scenarios = Generation_of_scenarios;

2) for $i=1$ to rows(Table_of_scenarios)

3) decision_optimization(Table_of_scenarios(i));

4) end

5) Table_of_scenarios $=$

Sort_by_present_value (Table_of_scenarios);

6) Best_Scenario = Table_of_scenarios(last_row);

7) Output(Best_Scenario);

Each step of this process will be described in the following subsections.

\subsection{Definition and representation of a scenario}

In our model we have four tree species oak, blockhead, stone pine, eucalyptus and four types of treeless land. Associated to each tree species we have four reforestation land when the trees become old. We have as well four types of treeless land. In the model proposed in [7] the land occupied by some type of tree can only be reforested by the same kind of tree. In our model we do not take into account this constraint. Then we can reforest a treeland piece that was occupied by oaks with stone pines. By other side, if we reforested some treeland piece with some type of tree, we cannot use this same type for reforesting another reforestation treeland piece. In what concerns the four types of utilizations of treeless land, we can repeat the utilization type in this kind of surfaces without restriction. Then a scenario is composed by three parts. The first part is associated with way we use the reforestation land, the second part is associated with way we use the treeless land, and third part is associated with the economic performance of the utilization of the land utilization of the reforestation land and the treeless land. The first vector position is associated with the oak reforestation land, the second vector position with the blockhead reforestation land, the third vector position is associated with stone pine reforestetion land, the fourth vector position with the eucalyptus reforestation land, etc. For representing a scenario in Matlab we use a numerical vector with nine places. The first four positions are used for the reforestation land, the following four positions for the treeless land, and the last position for the present value economic performance. We assigned numbers for codify each reforestation species as well as for each treeless utilization as follows:

For reforestation land

\begin{tabular}{|l|l|}
\hline species & code \\
\hline Oak & 2 \\
\hline blockhead & 4 \\
\hline stone pine & 6 \\
\hline eucalyptus & 8 \\
\hline
\end{tabular}

For treeless land

\begin{tabular}{|l|l|}
\hline use & code \\
\hline culture & 9 \\
\hline Pastureland & 10 \\
\hline scrub land & 11 \\
\hline other & 12 \\
\hline
\end{tabular}

So an instance of scenario could be

\begin{tabular}{|l|l|l|l|l|l|l|l|l|}
\hline 4 & 6 & 8 & 2 & 10 & 10 & 9 & 9 & $1,000,000$ \\
\hline
\end{tabular}

This vector means that the oak reforestation land will be occupied by blockhead, the blockhead reforestation land will be occupied by stone pines, the stone pine reforestation land will be occupied by culture, the culture reforestation land will be occupied by oaks, the scrub land as well by the pastureland, the pastureland will be used for pastureland and the culture land and the scrub land will be used for culture. The last number means that the present value profit of this scenario is 1,000, 000 Euros.

\subsection{Generation of the table of all possible scenarios}

Sometimes we have to face problems where an exhaustive exploration of all the possible cases in the state spaces is necessary and not only to calculate the total number of possible configurations in such state space. For example, looking for permutations of a given set of objects. This kind of tasks are called by some authors as enumeration problems but this term can be mistaken as the calculation of the number of possibilities and for this reason it is more appropriate to call them listing problems. Such is the kind of problem that we need to face for the generation of all possible scenarios of initial conditions in the land distribution of the model. In this section the generation of all the possible scenarios to be evaluated will be 
described. For this purpose we will have to generate a table whose lines will be numerical vectors of 9 positions where the 4 first positions represent the assignment of tree species to areas for artificial reforestation, the next 4 positions will correspond to the assignment of types of crops to areas of non-forest use and the last position will be used to record the evaluation of the cost at present value of said area allocation. In the case of the available areas used for reforestation, if one area is occupied by a species of tree, then the other areas that remain available cannot be used to reforest with the same species of tree, that is, in this case we will be talking about permutations. no repetition. On the other hand, in the areas for agricultural use, we can repeat crops, that is, we will be talking about permutations with repetition. The number of different ways of occupation of the reforestation land can be calculated as the number of permutations without repetition of the four tree species.

The concepts of combinatorial analysis that we will use to generate the different scenarios of land use and reforestation are those of the rule of product, permutations without repetition and combinations with repetition. We will define these concepts below that are taken from [14].

Definition 1. (The combinatorial rule of product): If an action of choice can be split into a first and a second independent stages, and if there are $m$ possible outcomes for the first stage and if, for each of these outcomes, there are n possible outcomes for the second stage, then the total procedure can be carried out, in the designated order, in $m \times n$ ways.

This principle is sometimes called the principle of choice. Based on the definition 11 we can be define the following concepts.

Definition 2. (Variations): $A$ variation of the $k$-th class of $n$ elements is an ordered k-element group formed from a set of $n$ elements. The elements are not repeated and depend on the order of the group's elements (therefore arranged).

$$
\begin{aligned}
& V_{k}(n)=n \times(n-1) \times(n-2) \times \\
& \cdots \times(n-k+1)=\frac{n !}{(n-k) !}
\end{aligned}
$$

Definition 3. (Permutation with repetitions): Same as the definition 2 but when the objects can be repeated. The calculation of $n$ objects in $k$ possible positions in a linear array (using the definition [1) is

$$
P R(n, k)=\underbrace{n \times n \times n \times \ldots \times n}_{k}=n^{k}
$$

Details about such notions of combinatorial analysis can be found in chapter 1 of book [14].
Thus, we can calculate the total number of possible permutations without repetition of $n$ objects making use of the formula of definition 2 .

In our case $n=4$ and $k=4$. So the number of possible land uses for reforestation would be $V_{4}(4)=$ 24.

In addition, the number of different ways of occupation of the treeless land can be calculated as the number of permutations with repetitions of $n$ objects taken from $k$ to $k$ using the formula of definition 3 .

In our case $n=4$ and $k=4$. So the number of possible land uses for non reforestation utilization of the land is $4^{4}=256$. From all the above and applying the product combinatorial principle, we have that the number of possible scenarios and therefore the number of rows in the table will be $24 \times 256=6144$. Once we know the total number of possible scenarios that correspond to the rows, we can proceed to the generation of the 6144 rows of the table. Combinatorial analysis allows us to be able to calculate the size that a problem space can have. However, sometimes this is not enough but it is necessary to explicitly obtain the complete list of these possibilities. That is exactly the problem that concerns us.

To do this, we first generate the list of all the permutations without repetition of the 4 numbers that correspond to the codes used to denote each species of tree. For this, a Matlab program inspired by the Lexicographic order permutation generation algorithm that can be found in [16] (page 319), was implemented. The algorithm such as it is described in in [16] (page 319) is

- L1.[Visit] Visit the permutation $a_{1} a_{2} \ldots a_{n}$

- L2.[Find $j$ ] Set $j \leftarrow n-1$. If $a_{j} \geq a_{j+1}$ decrease $j$ by 1 repeatedly until $a_{j}<a_{j+1}$. Terminate the algorithm if $j=0$.

- L3.[Increase $a_{j}$ ] Set $l \leftarrow n$.If $a_{j} \geq a_{l}$ decrease $l$ by 1 repeatedly until $a_{j}<a_{l}$. Then swap $a_{j} \leftrightarrow$ $a_{l}$.

- L4.[Reverse $\left.a_{j+1} \ldots a_{n}\right]$ Set $k \leftarrow j+1$ and $l \leftarrow$ $n$. Then while $k<l$ interchange $a_{k} \leftrightarrow a_{l}$ and set $k \leftarrow k+1, l \leftarrow l-1$. Return to $\mathbf{L 1}$

The algorithm correction is assured as step $\mathbf{L} \mathbf{2}$ has in the index $j$ the smallest index such that it has been visited to this point of processing all possible permutations starting with the prefix $a_{1} a_{2} \ldots a_{j}$ such that the following permutation in lexicographic order will make $a_{j}$ to have a bigger value, in $\mathbf{L} \mathbf{3}$ before the interchange $a_{j} \leftrightarrow a_{l}$ we have that $a_{j+1} \geq \ldots \geq a_{l-1} \geq$ $a_{l}>a_{j} \geq a_{l+1} \geq \ldots \geq a_{n}$ and after the interchange we have $a_{j+1} \geq \ldots \geq a_{l-1} \geq a_{j}>a_{l} \geq a_{l+1} \geq$ $\ldots \geq a_{n}$. According to D. Knuth in his book [16] this 
algorithm dates back to the 14th century in India, was proposed by Narayana Pandita at that time and has been rediscovered in other periods of the past such as in the preface of the book Specimen Analyticum by Lineis Curvis Ordinis by C.F. Rüdiger (Leipzig 1748). There are other more efficient algorithms to solve the problem of listing all possible permutations without repetition inspired by algorithms for obtaining n-tuples of Gray codes one of which is the algorithm of Adjacent Interchanges whose detailed description can be found at [16] page 320 , as well as another algorithm called Algorithm P(plain changes) [16], page 321 which originates from the programming of the Church Steeples in England in the 17th century (1653) (Cambridge Forty-eight, Ernest Morris The History and Art of Change Ringing (1931), R. Duckworth and F. Stedman, Tintinnalogia(1668)). There are many algorithms for generating the list of permutations without repetition, one of which can be known as (Plain changes) or Algorithm $\mathbf{P}$ and that can be consulted on page 322 of [16]. This algorithm dates from the 17 century in England and was used by workers in church steeples to program the melodies in a more varied way from generating all the possible permutations of the 5 bells that the church towers had.

Regarding the generation of the list of the different forms of occupation of the unfolded land, which we represent in the positions 5 to 8 of our row vector of 9 positions that make up each row of the aforementioned table, the situation is much simpler when dealing with permutations with repetition. The generation of this list of permutations with repetition in lexicographic order of 4-tuples of 4 possible positions is a very simple algorithm since it would consist of nested cycles in the following way

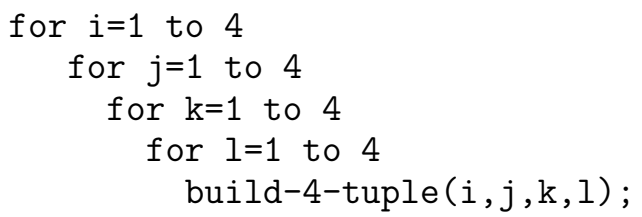

Once the $4^{4}$ possible 4 -tuples of positions have been generated, we choose the elements of the set of codes associated with the ungrounded land uses, which in this case are the values $9,10,11,12$ and with these values are filled the positions 5 to 8 of the row vector of the table. For the purposes of our forestry application it is sufficient to generate the list of possible permutations in lexicographical order. However it is interesting to mention that other forms of generation of $n$-tuples have been studied and are addressed in chapter 7 , subsection 7.2 of the book [16]. Some of these algorithms are related to Gray codes where it is sought that the $n$-tuples generated follow an order such that between one $n$-tuple and the next they only differ by a single element as shown in the following sequence of 4-tuples in binary:

$\begin{array}{llll}0000 & 0001 & 0011 & 0010\end{array}$

$\begin{array}{llll}0110 & 0111 & 0101 & 0100\end{array}$

$\begin{array}{llll}1100 & 1101 & 1111 & 1110\end{array}$

$\begin{array}{llll}1010 & 1011 & 1001 \quad 1000\end{array}$

Such codes have application in the conversion of ana$\log$ information to digital format and vice versa since they minimize the errors that can be generated in said format conversions. This type of coding was used in color television broadcasting and pulse modulation applications as well as in the analog transmission of digital signals. Many variants of Gray codes and their applications can be found in chapter 7 of the book [16].

\subsection{Sorting the table and selection of the best scenario}

Once all the possible values of the 8 first positions of the row vector of the table have been generated, we proceed to evaluate each of the scenarios corresponding to each row to fill the 9 position of the row that corresponds to the economic evaluation at present value of said stage. It is important to mention that some of these scenarios may violate the restriction of the total land availability and that is why we submit the table to a filter of these cases to eliminate them. Once this purification has been carried out, we order the table in ascending order with respect to the economic evaluation obtained. To do this, we apply a standard algorithm for sorting by selection and after this we will have in the last position of the table the option or scenario whose economic evaluation was the highest. Inglés

We should mention that the selection sort algorithm has a temporary performance in the worst case of $O\left(n^{2}\right)$ where $n$ is the number of rows to sort. This algorithm is efficient enough to sort the table, however for later versions we could use a more efficient sorting method whose temporal performance is of the order of $O(n \log (n))$ as is the case of Merge-Sort or the Quicksort algorithm.

\section{Results}

We carry out our simulation by calling the dynamic optimization routine for each row of our table of all possible scenarios for assigning uses of land, both wooded and bare. The time window used was $T=$ 100 , annual rate $r=0.03$ and with these parameters we call our simulator 6144 times that correspond to all the possible scenarios that we generate. Once this is done, we display the graphs corresponding to the best scenario and the worst scenario for comparison purposes. The results are shown in the following graphs. The starting land occupation $(t=0)$ for each call to the dynamic optimization routine is: 

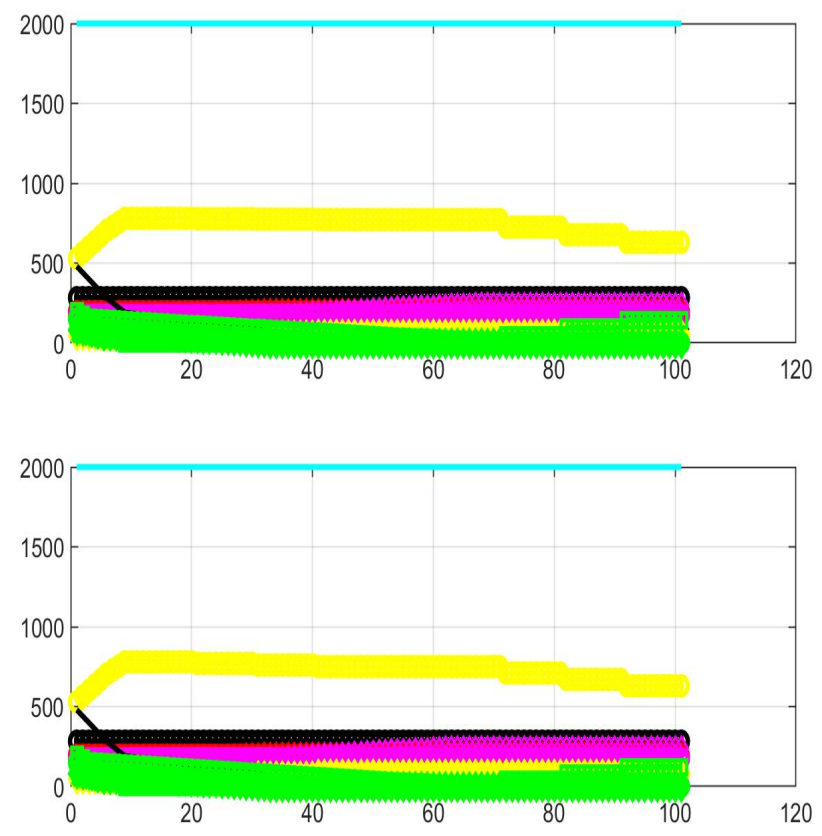

Figure 1: Worst and Best scenario. The blue line is the total surface, black line represent Oak, red line represent Stone pine, magenta line represent Blockhead, yellow line represent Eucalyptus and green represent pasture land.

\begin{tabular}{|c|c|}
\hline Land Use & Surface in Ha \\
\hline Natural Oak tree & 283 \\
\hline Artificial Oak tree & 85 \\
\hline Natural blockhead tree & 174 \\
\hline Artificial blockhead tree & 88 \\
\hline Natural stone pine tree & 195 \\
\hline Artificial stone pine tree & 120 \\
\hline Natural Eucalyptus tree & 45 \\
\hline Artificial Eucalyptus tree & 530 \\
\hline culture & 200 \\
\hline Pastureland & 150 \\
\hline scrub land & 80 \\
\hline other & 50 \\
\hline
\end{tabular}

After having carried out the evaluation of each scenario assigned to each row of the table, the results shown are shown in the following figures.

\section{Discussion and conclusions}

In this article we propose a mathematical agroforestry optimization model and from this we implement a simulation in MATLAB. The model works with four species of trees and four types of agricultural land

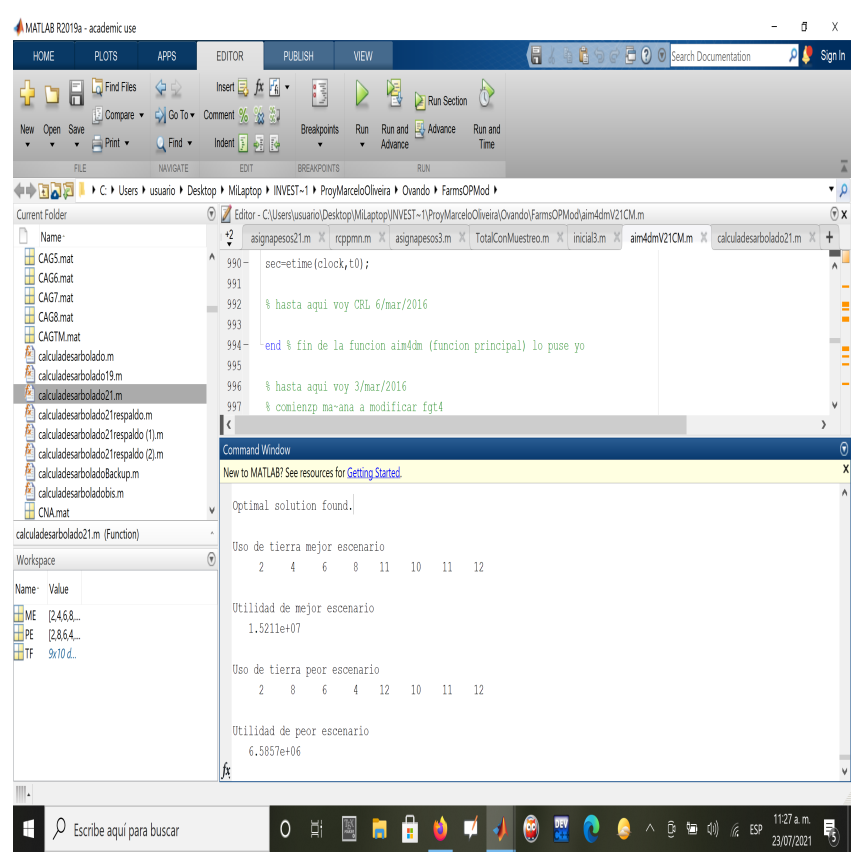

Figure 2: Screenshot of Best and Worst scenario obtained by the simulator

use and through the simulation we explore all possible combinations of agricultural land use for reforestation purposes to obtain the one that would be the most economically profitable. The simulation of each possible scenario took approximately three minutes and exploring all the possibilities represented having a laptop working for approximately a week. For this reason, we found it necessary to take a significant sample of all possible cases. Despite this, the time to process all the cases in the sample took around 48 hours of continuous processing. For this reason, we are considering the possibility that in future work we will make use of parallel programming to try to reduce processing time even more. Another possibility for future work is to try to apply other combinatorial optimization techniques to try to reduce the number of cases to be explored.

\section{Acknowledgments}

Paola Ovando acknowledges the support of the European Commission under the Marie Curie Intra-European Fellowship Programme (PIEF-2013621940). Carlos Rodríguez Lucatero and Marcelo Olivera Roel want to acknowledge the support given by the Universidad Autónoma Metropolitana Unidad Cuajimalpa. 


\section{Appendix}

\subsection{State variables of David Martín Barroso model}

This model was presented in the Phd. Thesis of Dr. David Martín Barroso in at the University Complutense of Madrid from Spain in the year 2003 [7]. The state variables of this model were

$$
\begin{gathered}
x_{t}=\left(\begin{array}{c}
x_{0, t} \\
\vdots \\
x_{320, t}
\end{array}\right) x_{a, t}=\left(\begin{array}{c}
x_{0, t}^{a} \\
\vdots \\
x_{320, t}^{a}
\end{array}\right) \\
s_{t}=\left(\begin{array}{c}
s_{0, t} \\
\vdots \\
s_{197, t}
\end{array}\right) s_{a, t}=\left(\begin{array}{c}
s_{0, t}^{a} \\
\vdots \\
s_{197, t}^{a}
\end{array}\right) \\
d_{t}=\left(\begin{array}{c}
d_{1, t} \\
d_{2, t} \\
d_{3, t}
\end{array}\right) \forall t \in\{0,1, \ldots, T\}, t<\infty
\end{gathered}
$$

Where $x_{t}, x_{a, t}$ represent the surface amount of natural and artificial generated Holm oak, $s_{t}, s_{a, t}$ represent the surface amount of natural and artificial generated blockhead, $d_{1, t}$ cereal cultivated surface, $d_{2, t}$ pastureland, and $d_{3, t}$ scrub surface.

\subsection{Control vectors of David Martín Barroso model}

The decisions taken by the agents involved in the agroforestal process are represented by control vector variables that influence the time evolution of the state variables as usual in any optimization model. This variables are called control vectors. There are six control variables, three correspond to the regeneration and reforestation decisions concerning the Holm oak, and the other three to the blockhead. They are mathematically represented as follows:

$$
\begin{aligned}
z_{t}=\left(\begin{array}{c}
n_{i, t}+n_{i, t}^{a} \\
0 \\
0 \\
\vdots \\
-n_{i, t} \\
\vdots \\
0 \\
0 \\
n_{s, t}+n_{s, t}^{a} \\
0 \\
0 \\
\vdots \\
-n_{s, t} \\
\vdots \\
0 \\
0
\end{array}\right) z_{a, t}=\left(\begin{array}{c}
t_{i, t} \\
0 \\
0 \\
\vdots \\
-n_{i, t}^{a} \\
\vdots \\
0 \\
0
\end{array}\right) \\
v_{t}=\left(\begin{array}{c}
t_{s, t} \\
0 \\
0 \\
\vdots \\
-n_{s, t}^{a} \\
\vdots \\
0 \\
0
\end{array}\right)
\end{aligned}
$$

Where $n_{i, t}$ represent de Holm oak surface naturally regenerated by natural planted trees of such species, $n_{i, t}^{a}$ represent de Holm oak surface naturally regenerated by artifially planted trees of such species, $t_{i, t}$ represent the surface reforested with Holm oak by humans. The variables $n_{s, t}, n_{s, t}^{a}$ and $t_{s, t}$ represent the corresponding surfaces for the blockhead. The negative entries correspond to the amount of trees that have achieved their productive age. In what concerns the dismasted land uses, the calculations depend on the cases of study and because of that the calculations are ad-hoc. In his thesis Dr. David Martin Barroso [7] talks about four specific farms $(j=1, \ldots, 4)$. The corresponding vectors are:

$$
u_{t}= \begin{cases}\left(\xi_{t},-t_{i, t}, 0\right) & \text { for } j=1 \\ \left(0,-\left(t_{i, t}+t_{s, t}\right), \xi_{t}\right) & \text { for } j=2 \\ \left(\xi_{t},-\left(t_{i, t}+t_{s, t}\right), 0\right) & \text { for } j=3 \\ \left(0,\left(\xi_{t}-t_{i, t}\right), t_{s, t}\right) & \text { for } j=4\end{cases}
$$

Where $\xi_{t}$ is a transition function of soil utilization and is calculated as follows

$$
\xi_{t}= \begin{cases}x_{320, t-1}+x_{320, t-1}^{a} & \text { for } j=1 \\ x_{320, t-1}+x_{320, t-1}^{a}+s_{196, t}+s_{194, t}^{a} & \forall j \neq 1\end{cases}
$$

\subsection{The state vector movement law of David Martín Barroso model}

The movement law equations that describe the temporal evolution of the controled state variables are the following.

$$
\left.\begin{array}{l}
x_{t+1}=T x_{t}+z_{t+1} \\
x_{a, t+1}=T x_{a, t}+z_{a, t+1} \\
s_{t+1}=A_{n} s_{t}+v_{t+1} \\
s_{a, t+1}=A_{a} s_{a, t}+v_{a, t+1} \\
d_{t+1}=d_{t}+u_{t+1}
\end{array}\right\}=\forall t \in\{0, \ldots, T-1\}, T<\infty
$$

Where $T, A_{n}$ and $A_{a}$ are transition matrix where all the elements are equal to zero excepted the elements just under the main diagonal whose values are equal to 1 . The dimensions of $T$ are $321 \times 321, A_{n}$ are $197 \times 197$ and $A_{a}$ are $195 \times 195$. 


\subsection{The control variable constrains of David Martín Barroso model}

In [7] they assume that the farm land owners don't change the size of their terrains by means of comercial transactions in such a way that the total surface of each farm stay constant along the time. This global resource constrain can be expresed by the following equation

$$
S_{j}=i(321) \cdot\left(x_{t}+x_{a, t}\right)+i(197) \cdot s_{t}+i(197) \cdot s_{a, t}+i(3) \cdot d_{t}
$$

where $S_{j}$ represent the total surface of the farm $j \in$ $\{1, \ldots, 4\}$, for $t \in\{1, \ldots, T\}$, and $i(n)$ is a row vector of $n$ ones.

The author of the model in [7] also assumes some non negativity constrains on the control variables expressed mathematically as follows

$$
n_{i, t}, n_{i, t}^{a}, n_{s, t}, n_{s, t}^{a}, t_{i, t}, t_{s, t} \geq 0
$$

Additionally some constrains regarding the natural regeneration process are imposed in such a way that the surface to be regenerated cannot be greater than the surface of trees in crital age. That is expressed as follows:

\subsection{Objective function of David Martín Barroso control optimization model}

The objective function of a control optimization model represents the base on which a deciding agent takes decisions. In the case of this agroforestry problem the function whose value is maximized is a cubic polynomial that evaluates the profit net present value of the reforested land depending on the soil utilization. This function has the following mathematical form:

$$
\begin{aligned}
f_{i}= & a_{i} \tau^{3}+b_{i} \tau^{2}+c_{i} \tau+d_{i} \\
& \forall \tau \in\{0, \ldots, 320\} \text { Holm oak } \\
f_{s}= & a_{s} \tau^{3}+b_{s} \tau^{2}+c_{s} \tau+d_{s} \\
& \forall \tau \in\{0, \ldots, 196\} \text { blockhead }
\end{aligned}
$$

Based on these functions it can be calculated the land price for hectare row vectors for each type of plantation and stored in the following vectors:

$$
\begin{aligned}
& f_{i}=\left(f_{i}(0), \ldots, f_{i}(320)\right) \\
& f_{s}=\left(f_{s}(0), \ldots, f_{s}(196)\right) \\
& f_{s, a}=\left(f_{s}(0), \ldots, f_{s}(196)\right) \\
& f_{d}=\left(p_{c t}, p_{p}, p_{m}\right)
\end{aligned}
$$

For enabling the agents to take decisons, it can be calculated the level of annual return vectors using diferent costing methods for each kind of soil utilization. The following row vectors store this information:

$$
\begin{aligned}
& y^{i, n}=\left(y_{0}^{i, n}, \ldots, y_{320}^{i, n}\right) \\
& y^{i, a}=\left(y_{0}^{i, a}, \ldots, y_{320}^{i, a}\right) \\
& y^{s, n}=\left(y_{0}^{s, n}, \ldots, y_{196}^{s, n}\right) \\
& y^{s, a}=\left(y_{0}^{s, a}, \ldots, y_{194}^{s, a}\right) \\
& y^{s, d}=\left(y_{c t}^{s}, y_{p}^{s}, y_{m}^{s}\right)
\end{aligned}
$$

Assuming that the deciding agent maximizes the finite horizon net present value of the profit associated to the average hectare of the farm $j$, the objective function gets the following form:

$$
\begin{aligned}
F_{j}= & \frac{1}{S_{j}}\left\{\sum _ { t = 0 } ^ { T } \delta ^ { t } \left[y^{i, n} x_{t}\right.\right. \\
& \left.+y^{i, a} x_{a, t}+y^{s, n} s_{t}+y^{s, a} s_{a, t}+y^{q, d} d_{t}\right] \\
& -\left[f_{i}\left(x_{0}+x_{a, 0}\right)+f_{s} s_{0}+f_{s, a} s_{a, 0}+f_{d} d_{0}\right] \\
& \left.+\delta^{T}\left[f_{i}\left(x_{T}+x_{a, T}\right)+f_{s} s_{T}+f_{s, a} s_{a, T}+f_{d} d_{T}\right]\right\} \\
& \quad q= \begin{cases}i \text { (ilex) } & \text { for } j=1,3 \\
s \text { (suber) } & \text { for } j=2,4\end{cases}
\end{aligned}
$$

\subsection{David Martín Barroso control optimization model}

Finally the control optimization problem can be written as the maximization of the objective function as follows:

$$
\max _{\left\{n_{i, t}, n_{i, t}^{a}, n_{s, t}, n_{s, t}^{a}, t_{i, t}, t_{s, t}\right\}_{t=1}^{t=T}} F_{j}
$$


subject to: equations (25) (26) (27)(28)(29) and the given intial conditions $x_{0}, x_{a, 0}, s_{0}, s_{a, 0}, d_{0}$ as well as the time horizon $T$.

\subsection{State Variables of our model first variation}

Based on the model mentioned in the last subsections our first variation on this model consited in adding one additional tree species, the Stone Pine tree. For this end we added the respective three decision adding the corresponding control variable vectors. We also have added more flexibility to the model of [7] by generalizing with variables the corresponding renovation tours for ecah species of trees. Fisrt of all the new set of state variables are represented mathematically as follows:

$$
\begin{array}{r}
x_{t}=\left(\begin{array}{c}
x_{0, t} \\
\vdots \\
x_{T_{1}, t}
\end{array}\right) x_{a, t}=\left(\begin{array}{c}
x_{0, t}^{a} \\
\vdots \\
x_{T_{2}, t}^{a}
\end{array}\right) \\
s_{t}=\left(\begin{array}{c}
s_{0, t} \\
\vdots \\
s_{T_{3}, t}
\end{array}\right) s_{a, t}=\left(\begin{array}{c}
s_{0, t}^{a} \\
\vdots \\
s_{T_{4}, t}^{a}
\end{array}\right) \\
p_{t}=\left(\begin{array}{c}
p_{0, t} \\
\vdots \\
p_{T_{5}, t}
\end{array}\right) p_{a, t}=\left(\begin{array}{c}
p_{0, t}^{a} \\
\vdots \\
p_{T_{6}, t}^{a}
\end{array}\right) \\
d_{t}=\left(\begin{array}{c}
d_{1, t} \\
d_{2, t} \\
d_{3, t}
\end{array}\right) \forall t \in\{0,1, \ldots, T\}, t<\infty
\end{array}
$$

\section{References:}

[1] Azqueta, D. Introducción a la economiía ambiental. McGraw-Hill Madrid 2002.

[2] Bielsa, I.; Pons, X.; Bunce, B. Agricultural abandonment in the north eastern Iberian Peninsula: The use of basic landscape metrics to support planning. Journal of Environmental Planning and Management 2005, 48(1), 85-102. https://doi.org/10.1080/0964056042000308166

[3] Blot, J.; Hayek,N. Infinite-horizon optimal control in the discrete-time framework. New York: Springer. (2014)

[4] Campos, P.; Ovando P.; Mesa B.; Oviedo J. L. Environmental Income of Live- stock Grazing on Privately-owned Silvopastoral Farms in Andalusia, Spain. Land Degra- dation and Development. 2016.
[5] Cerdá, E.; Martín-Barroso, D. Optimal control for forest management and conservation analysis in dehesa ecosystems. European Journal of Operational Research (2013). https://doi.org/10.1016/j.ejor.2012.12.010

[6] Crow, T. R., Host, G. E.; Mladenoff, D. J. Ownership and ecosystem as sources of spatial heterogeneity in a forested landscape, Wisconsin, USA. Landscape Ecology (1999), 14(5), 449-463. https://doi.org/10.1023/A:1008084123874

[7] Martínez Barrosso D. Instrumentos de análisis económico en sistemas agroforestales. $P h D$ Thesis, Universidad Complutense de Madrid 2003.

[8] https://ec.europa.eu/web/forestry, 2013

[9] http://eur-nexo/legalcontent/ES/TXT/? uri=LEGISSUM:160040, (revised January 2018), 2018

[10] Faustmann, M. The Faustmann Model (part 1) Introduction to Forestry,Forest Policy, and Economics. web.archive.org/web/20111229211645/ 2011.

[11] Flichman G.; Allen T. Bio-economic modeling:State-of-the-art and key priorities. agris.fao.org 2015.

[12] Gil-Tena, A.; Saura, S.; Brotons, L. Effects of forest composition and structure on bird species richness in a Mediterranean context: Implications for forest ecosystem management. Forest Ecology and Management (2007), 242(2-3), 470-476. https://doi.org/10.1016/J.FORECO.2007.01.080

[13] Gómez-Limón, J.; De Lucío Fernández, J. V. Changes in use and landscape preferences on the agricultural-livestock landscapes of the central Iberian Peninsula (Madrid, Spain). Landscape and Urban Planning (1999), 44(4), 165-175. https://doi.org/10.1016/S01692046(99)00020-1

[14] Grimaldi R.P. Discrete and Combinatorial Mathematics: An Applied Introduction Third Edition Addison-Wesley 1994.

[15] Kienast, F.; Frick, J.; van Strien, M. J.; Hunziker, M. The Swiss Landscape Monitoring Program - A comprehensive indicator set to measure landscape change. Ecological Modelling (2015), 295, 136-150. https://doi.org/10.1016/j.ecolmodel.2014.08.008 
[16] Knuth D. The art of Computer Programming: Combinatorial Algorithms, Part 1, Volume 4A, Addison-Wesley, 1997

[17] Lasanta-Martínez, T.; Vicente-Serrano, S. M.; Cuadrat-Prats, J. M. Mountain Mediterranean landscape evolution caused by the abandonment of traditional primary activities: A study of the Spanish Central Pyrenees. Applied Geography (2005), 25(1), 47-65. https://doi.org/10.1016/j.apgeog.2004.11.001

[18] Lasanta, T.; Beguería, S.; García-Ruiz, J. M. Geomorphic and Hydrological Effects of Traditional Shifting Agriculture in a Mediterranean Mountain Area, Central Spanish Pyrenees. Mountain Research and Development (2006), 26(2), 146-152. https://doi.org/10.1659/02764741(2006)26[146:GAHEOT]2.0.CO;2

[19] Newman D.H. The Optimal Forest Rotation: A Discussion and Annotated Bibliography. Technical Report USDA-Forest Service (1988).

[20] Pyy J., Ahtikoski A., Laitinen E., Siipilehto J. Introducing a Non-Stationary Matrix Model for Stand-Level Optimization, an Even-Aged Pine (Pinus Sylvestris L.) Stand in Finland forests, MDPI Open Journal,http://www.mdpi.com/journal/forests, Forests 2017, 8, 163; doi:10.3390/f8050163

[21] Pyy J., Ahtikoski A., Lapin A., Laitinen E. Solution of Optimal Harvesting Problem by Finite Difference Approximations of SizeStructured Population Model Mathematical an Computational Applications, MDPI Open Journal, http://www.mdpi.com/journal/mca, Math. Comput. Appl. 2018, 23, 22; doi:10.3390/mca23020022

[22] Ryan, M.;C. ODonoghe; Phillips, H. Modelling Financially Optimal Afforestation and Forest Management Scenarios Using a Bioeconomic Model, Open Journal of Forestry (2016), 06(01), 19-38.

[23] Vicente-Serrano, S. M.; Lasanta, T.; Cuadrat, J. M.; Cuadrat, J. M. Transformaciones en el paisaje del Pirineo como consecuencia del abandono de las actividades económicas tradicionales. Pirineos (2000), 155(0), 111-133. https://doi.org/10.3989/pirineos.2000.v155.91

[24] Ovando, P., Beguería, S., and Campos, P. Carbon sequestration or water yield? The effect of payments for ecosystem services on forest management decisions in Mediterranean forests. Water Resources and Economics, Elsevier, Volume 28, October 2019, 100119,https://doi.org/10.1016/j.wre.2018.04.002

[25] Ovando, P., P. Campos, B. Mesa, A. Alvarez, C. Fernández, J. Oviedo, A. Caparros, and B. Alvarez-Farizo Renta y capital de estudios de caso de fincas agroforestales de Andalucía. In P. Campos and P. Ovando (Eds.), Renta total y capital de las fincas agroforestales de Andalucía, pp. 156-445. Madrid: Memorias científicas de RECAMAN. Vol 4. Editorial CSIC.

\section{Contribution of individual authors to the creation of a scientific article (ghostwriting policy)}

Carlos Rodríguez Lucatero carried out the mathematical modelling as well as the MATLAB implementation of the simulator. He also contributed with the writing of the present article. Marcelo Olivera Villarroel contributed in the modelling of economic aspects as well as forestry aspects of the mathematical model on which the simulator is based. $\mathrm{He}$ also contributed with the writing of the present article. Paola Ovando contributed in the modelling of forestry aspects of the mathematical model as well as with the forestry data on which the simulator is based.

\section{Sources of funding for research presented in a scientific article or scientific article itself}

Paola Ovando acknowledges the support of the European Commission under the Marie Curie Intra-European Fellowship Programme (PIEF-2013621940). Carlos Rodríguez Lucatero and Marcelo Olivera Roel want to acknowledge the support given by the Universidad Autónoma Metropolitana Unidad Cuajimalpa.

\section{Creative Commons Attribution License 4.0 (Attribution 4.0 International, CC BY 4.0)}

This article is published under the terms of the Creative Commons Attribution License 4.0

https://creativecommons.org/licenses/by/4.0/deed.en US 